\title{
Caracterização do Comportamento Caótico da Variabilidade da Frequência Cardíaca (VFC) em Jovens Saudáveis ${ }^{1}$
}

M.T. FERREIRA², Programa de Mestrado em Biometria, Instituto de Biociências de Botucatu - IBB, UNESP, 18618-000 Botucatu, SP, Brasil.

M. MESSIAS, 3 , Departamento de Matemática, Estatística e Computação DMEC, Faculdade de Ciências e Tecnologia - FCT, UNESP, 19060-900 Presidente Prudente, SP, Brasil.

L.C.M. VANDERLEI ${ }^{4}$, C. M. PASTRE ${ }^{5}$, Laboratório de Fisiologia do Estresse Departamento de Fisioterapia, Faculdade de Ciências e Tecnologia - FCT, UNESP, 19060-900 Presidente Prudente, SP, Brasil.

Resumo. A análise da variabilidade da frequência cardíaca (VFC) é uma técnica utilizada na avaliação do comportamento do sistema nervoso autônomo (SNA) que tem emergido como uma medida simples e não-invasiva dos impulsos autonômicos. Neste trabalho utilizamos técnicas da teoria dos sistemas dinâmicos não-lineares, incluindo a teoria do caos, no estudo de séries temporais da frequência cardíaca de jovens saudáveis com o objetivo de caracterizar o possível comportamento caótico da VFC. Foram analisadas séries de intervalos RR de 10 voluntários saudáveis, na faixa etária de 18 a 22 anos. Os índices não-lineares calculados sugerem o possível comportamento caótico das séries analisadas.

Palavras-chave. Variabilidade da Frequência Cardíaca, séries temporais experimentais, comportamento caótico.

\section{Introdução}

Alterações na frequência cardíaca, definidas como variabilidade da frequência cardíaca (VFC), são normais e esperadas e indicam a habilidade do coração em responder aos múltiplos estímulos fisiológicos e ambientais, dentre eles, respiração, exercício físico, estresse mental, alterações hemodinâmicas e metabólicas, sono e

\footnotetext{
${ }^{1}$ Trabalho realizado com financiamento da CAPES.

2 mteodoraf@ibb.unesp.br

3 marcelo@fct.unesp.br

${ }^{4}$ vanderle@fct.unesp.br

${ }^{5}$ pastre@fct.unesp.br
}

Recebido em 09 Setembro 2009; Aceito em 31 Maio 2010. 
ortostatismo, bem como em compensar desordens induzidas por doenças [12]. Tratase de uma medida não invasiva que pode ser utilizada para identificar fenômenos relacionados ao sistema nervoso autônomo (SNA) [11].

Em condições normais, um ciclo cardíaco completo é representado pelas ondas P, Q, R, S e T (Figura 1(a)). A distância entre duas ondas R, em milissegundos, é denominada intervalo RR e a partir deles uma série temporal (Figura 1(b)) pode ser construída.

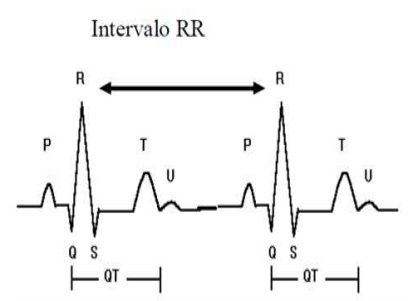

(a) Intervalo RR.

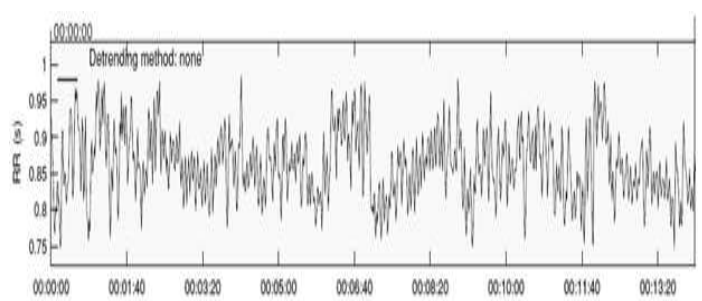

(b) Série Temporal formada por intervalos RR.

Figura 1: Exemplo de intervalo RR e a respectiva série temporal utilizada para análise da VFC.

A análise da VFC, por ser uma técnica não invasiva, constitui um procedimento valioso para a avaliação da função do SNA em muitas condições clínicas. O ramo simpático do SNA aumenta a frequência cardíaca, implicando em intervalos mais curtos entre os batimentos, com a consequente diminuição da VFC. Por sua vez, o ramo parassimpático diminui a frequência, resultando em intervalos maiores entre os batimentos e o aumento da VFC. Desse modo, alterações nos padrões da VFC constituem um indicador precoce e sensível do comprometimento da saúde. Em geral, uma alta VFC é sinal de boa adaptabilidade das funções do organismo, especialmente do SNA, indicando um indivíduo saudável, com os mecanismos de controle autonômicos funcionando bem. Por outro lado, uma baixa variabilidade é frequentemente indicadora de adaptabilidade anormal ou insuficiente do SNA, indicando a possível presença de mau funcionamento fisiológico.

Séries temporais da frequencia cardíaca são, em geral, estudadas por meio de métodos estatísticos, como os descritos em [2]. Porém, devido à sua complexidade, também têm sido estudadas do ponto de vista dinâmico, associado aos sistemas determinísticos não-lineares que apresentam comportamento caótico, como por exemplo a equação logística [4]. Tais sistemas apresentam um comportamento imprevisível a longo prazo, sendo caracterizados principalmente pela dependência sensível das soluções com relação às condições iniciais e pela presença de atratores estranhos.

Atualmente sugere-se na literatura $[1,4,6,14]$ que as séries temporais da frequência cardíaca associadas a indivíduos saudáveis apresentam comportamento caótico, enquanto que séries associadas a indivíduos com algum distúrbio cardíaco ou outro 
problema de saúde apresentam perda de complexidade, indicada pela diminuição no valor de índices relacionados à dinâmica não-linear calculados para a VFC, como por exemplo, o expoente de Lyapunov.

A análise de séries temporais experimentais obtidas da frequência cardíaca por métodos não-lineares vem ganhando crescente interesse, pois existem evidências de que os mecanismos envolvidos na regulação cardiovascular provavelmente interagem entre si de modo não-linear [1].

Além disso, em $[1,6,7,14]$ os autores afirmam que índices não-lineares fornecem evidências de que a dinâmica fundamental da frequência cardíaca é não-linear e propõem a possibilidade de existência de caos determinístico relacionado a mesma.

Levando em consideração os aspectos acima, neste trabalho buscamos caracterizar a possível existência de comportamento caótico na VFC de jovens saudáveis. $\mathrm{Na}$ seção 2, descrevemos os métodos não-lineares utilizados neste trabalho para a análise das séries temporais. Na seção 3, apresentamos a casuística e o método de coleta dos dados. Já, na seção 4, descrevemos os resultados obtidos, que são discutidos na seção 5 . Na seção 6 apresentamos as conclusões.

\section{Métodos Não-lineares de Análise de Séries Tem- porais}

A seguir, apresentamos os índices utilizados neste trabalho para a caraterização das séries temporais da VFC de jovens saudáveis.

\section{Função de Autocorrelação.}

A função de autocorrelação $A(\tau)$ de uma dada série temporal $x_{t}$ é definida por

$$
A(\tau)=\frac{1}{N-\tau} \sum_{t=1}^{N-\tau} \frac{\left(x_{t}-\bar{x}\right)\left(x_{t+\tau}-\bar{x}\right)}{\sigma^{2}},
$$

onde $\mathrm{N}$ é o número de pontos da série, $\bar{x}$ sua média e $\sigma^{2}$ sua variância. Esta função indica por quanto tempo o valor da série no instante $t$ depende de seus valores prévios. Em outras palavras, $A(\tau)$ mede o grau de semelhança existente na série à medida que o tempo passa. Para séries temporais com comportamento caótico, $A(\tau) \rightarrow 0$ quando $\tau \rightarrow \infty$, ou seja, a semelhança da série consigo mesma diminui com o tempo e acaba por desaparecer completamente [4].

\section{Gráfico de Poincaré.}

Neste gráfico cada intervalo RR é plotado como uma função de seus intervalos RR anteriores [1, 14], ou seja, plota-se o par ordenado $\left(x_{t}, x_{t+1}\right) \operatorname{com} t=1,2 \ldots N-1$, no plano cartesiano. É uma técnica dinâmica não-linear que retrata a natureza das flutuações dos intervalos RR das séries temporais. A análise do gráfico de Poincaré pode ser feita qualitativa ou quantitativamente. Qualitativamente, a forma do gráfico pode indicar o grau de falha cardíaca. Quantitativamente, ajusta-se uma elipse aos pontos do gráfico, com centro determinado pela média dos intervalos RR. 
Pode-se assim calcular o índice SD1, que mede o desvio padrão das distâncias dos pontos à diagonal $y=x$, e o índice SD2 que mede o desvio padrão das distâncias dos pontos à reta $y=-x+R R_{m}$, onde $R R_{m}$ é a média dos intervalos $\mathrm{RR}$. O índice SD1 reflete a variabilidade instantânea de batimento-a-batimento da frequência cardíaca e representa a atividade parassimpática, enquanto que o índice SD2 reflete a variabilidade contínua de batimento-a-batimento e representa a variabilidade global $[1,6,14]$.

\section{Reconstrução do Espaço de Fase.}

A reconstrução do espaço de fase tem provado ser uma ferramenta poderosa na análise de séries temporais experimentais. A idéia básica desta reconstrução está calcada no fato de que a história temporal de uma série contém informações sobre variáveis de estado não observáveis que podem ser usadas para determinar um estado presente. Uma série temporal experimental não mede todas as variáveis de estado do sistema e, usualmente, tem-se disponível a evolução no tempo de apenas uma variável de estado, representada por uma série temporal $x_{t}$, que representa a trajetória de um dado sistema dinâmico. A reconstrução do espaço de fase é baseada no Teorema da imersão de Takens (Takens embedding theorem) [10]. Este teorema permite reconstruir um espaço de fase $m$-dimensional similar ao espaço de fase original, a partir de uma única variável de estado, a variável medida. Este espaço reconstruído, apresenta uma suave variação de coordenadas em relação ao espaço original, preservando os invariantes geométricos do sistema, tais como a dimensão do atrator e os expoentes de Lyapunov.

Takens provou que, no espaço de fase formado pelos eixos

$$
\vec{\xi}_{x(t)}=(x(t), x(t+p), x(t+2 p), \ldots, x(t+(m-1) p)),
$$

o atrator reconstruído é topologicamente equivalente ao atrator "real", sobre o qual conhece-se apenas a evolução em tempo discreto da variável de estado. Na sua prova, Takens assumiu que a série é formada por infinitos pontos e que não há ruído. Segundo Takens, se essas condições são satisfeitas, as propriedades topológicas do atrator reconstruído são preservadas. Chamamos de espaço de imersão (embedding space) o espaço no qual realiza-se a reconstrução. Denomina-se $m$ de dimensão de imersão (embedding dimension) e $p$ de passo da reconstrução ou tempo de atraso (time delay).

Neste método o mais difícil é selecionar o tempo de atraso (ou o passo) e a dimensão de imersão adequados. De acordo com a literatura $[1,6,7,14]$ usa-se dimensão de imersão $m=3$ e passo $p=1$, no estudo da série da VFC.

\section{Plot de Recorrência.}

É usado no estudo da dependência temporal de uma série, ou seja, no estudo de sua estacionariedade [8]. Segundo Eckmann [3] por meio de um Plot de Recorrência é possível visualizar o comportamento de trajetórias no espaço de fase e ainda mostrar todos os tempos no qual um estado de um sistema dinâmico se repete. A repetição de estados é uma propriedade fundamental de um sistema dinâmico determinístico e é um comportamento típico em sistemas caóticos e não-lineares [4]. 
Um plot de recorrência é definido por uma matriz simétrica $[N-(m-1) p] \times[N-$ $(m-1) p]$ composta de zeros e uns. O elemento na i-ésima linha e j-ésima coluna da matriz $\mathrm{PR}$, isto é, $P R(i, j)$, é descrito por

$$
\operatorname{PR}(i, j)=\left\{\begin{array}{lll}
1 & \text { se } & d\left(\vec{\xi}_{i}-\vec{\xi}_{j}\right)<r \\
0 & \text { se } & C C
\end{array}\right.
$$

onde $d\left(\vec{\xi}_{i}, \vec{\xi}_{j}\right)$ é a distância euclidiana dos vetores reconstruídos e $r$ é um limiar fixo.

$\mathrm{Na}$ análise de séries temporais da VFC, para séries temporais de sujeitos saudáveis o $P R$ tem uma linha diagonal e menos quadrados aparentes, indicando VFC mais alta [8].

\section{Entropia Aproximada - ApEn.}

Pincus propôs este tipo de entropia como sendo a razão de geração de novas informações em um sistema, podendo ser aplicada tipicamente para séries temporais de dados clínicos curtos e com ruído.

A ApEn detecta as mudanças ocorridas em uma série temporal experimental e fornece um número não negativo para a série, com valor maior correspondendo à maior complexidade ou irregularidade nos dados [1, 14].

\section{Entropia da Amostra - SampEn.}

Richman e Randall [4] desenvolveram e caracterizaram a SampEn, que é uma valiosa medida da regularidade de uma série temporal. A SampEn é muito similar a $A p E n$, mas há uma diferença computacional entre ambas.

A importância deste índice na análise de séries temporais da VFC reside no fato de que ela é uma medida da desordem existente na série, apresentando valores maiores para os sinais cardíacos de sujeitos saudáveis e valores menores para sinais cardíacos de sujeitos com alguma deficiência cardíaca $[1,6]$.

\section{Detrended Fluctuation Analysis - DFA.}

O índice DFA é usado para quantificar a propriedade fractal de séries temporais de intervalos RR, sendo utilizado para a detecção de possíveis anormalidades presentes em um sujeito, baseando-se em coeficientes $\alpha$. Neste trabalho apresentamos dois expoentes $\alpha_{1}$ e $\alpha_{2}$, sendo que $\alpha_{1}$ está relacionado com as flutuações de tempo curto da VFC e $\alpha_{2}$ com as flutuações de tempo longo. Na análise de séries temporais da VFC, para sujeitos saudáveis, o valor de $\alpha_{1}$ fica próximo de 1 e é maior do que o valor de $\alpha_{2}$, conforme [14].

\section{Dimensão de Correlação.}

A dimensão de corrrelação representa a dispersão ou densidade de um atrator em um espaço de fase, sendo pois, uma medida da complexidade do processo vital investigado e representa uma das formas mais populares de se calcular a dimensão do atrator neste espaço $[7,14]$. De acordo com a literatura, o seu valor será alto para séries RR caóticas e diminuirá quando a variação da série tornar-se menor ou rítmica, indicando baixa $\mathrm{VFC}[1,7,14]$. 


\section{Expoente de Lyapunov.}

Este índice tem se mostrado uma ferramenta bastante útil de diagnóstico dinâmico de sistemas caóticos e pode também ser usado para o cálculo de outras quantidades invariantes como, por exemplo, a dimensão do atrator. Os expoentes de Lyapunov, denotados por $\lambda_{i}$, quantificam a dependência do sistema em relação às condições iniciais. Assim, este expoente é uma medida da razão na qual trajetórias separam-se umas das outras, sendo usado como uma medida da previsibilidade do processo. Um expoente positivo implica que as órbitas estão sobre um atrator caótico, assim, a existência de pelo menos um expoente de Lyapunov positivo para um certo sistema indica que este é caótico. Para estabelecer se um processo dinâmico é caótico, é suficiente calcular o maior dos expoentes de Lyapunov pois, desta forma, é possível saber se trajetórias vizinhas divergem ou não $[1,4,7,14]$.

\section{Conjectura de Kaplan-York.}

Em 1979 J. L. Kaplan e J. A. York propuseram uma conjectura a respeito da relação entre os expoentes de Lyapunov e a dimensão do atrator de um sistema dinâmico ou série temporal experimental [4]. A dimensão calculada a partir dos expoentes de Lyapunov é muitas vezes chamada de dimensão de Kaplan-York e é dada pela equação

$$
D_{K Y}=j+\frac{\sum_{i=1}^{j} \lambda_{i}}{\left|\lambda_{j+1}\right|}
$$

onde os $\lambda_{i}$ são os expoentes de Lyapunov ordenados de forma decrescente e $j$ é o maior inteiro tal que $\sum_{i=1}^{j} \lambda_{i}>0$.

No cálculo dos índices não-lineares descritos acima, para as séries RR de jovens saudáveis, utilizamos os softwares: HRV Analysis, do Biomedical Signal Analysis Group, disponível no endereço http://kubios.uku.fi/KubiosHRV/; e TISEAN - Time Series Analysis, disponível no site http://www.mpipks-resden.mpg.de/tisean/. Os gráficos foram feitos no MATLAB.

\section{Casuística e Método de Coleta dos Dados}

Para a realização deste trabalho foram analisadas as séries temporais de 10 voluntários considerados saudáveis. Na Tabela 1 apresentamos a média seguida do respectivo desvio-padrão da idade, peso, altura e frequência cardíaca (FC) dos voluntários estudados.

Os voluntários foram devidamente informados sobre os procedimentos e objetivos do estudo e, após concordarem, assinaram um termo de consentimento livre e esclarecido. O procedimento experimental foi realizado em sala com temperatura entre $21^{\circ} \mathrm{C}$ e $23^{\circ} \mathrm{C}$ e umidade entre $40 \%$ e $60 \%$.

Foram colocados, no tórax dos voluntários, a cinta de captação e, no seu pulso, o receptor de frequência cardíaca Polar S810i, equipamento previamente validado para captação da frequência cardíaca batimento a batimento e a utilização da série temporal obtida para análise da VFC $[9,13]$. 
Tabela 1: Valores médios, seguidos dos respectivos desvios padrões, da idade (em anos), do peso (em $\mathrm{Kg}$ ), da altura $(\mathrm{em} \mathrm{cm}$ ) e da frequência cardíaca (em batimentos por minuto - bmp) dos 10 voluntários tomados como amostra total deste trabalho.

\begin{tabular}{|c|c|c|c|}
\hline Idade (em anos) & Peso $(\mathrm{em} \mathrm{Kg})$ & Altura $(\mathrm{em} \mathrm{cm})$ & FC (em bpm) \\
\hline \hline $20,2 \pm 1,31$ & $56,09 \pm 9,70$ & $166,25 \pm 8,35$ & $83,4 \pm 7,58$ \\
\hline
\end{tabular}

Após a colocação da cinta e do monitor os voluntários foram colocados deitados em posição supina e permaneceram em repouso em respiração espontânea por 20 minutos. Para a análise da VFC, o padrão de seu comportamento foi registrado batimento a batimento durante todo o protocolo experimental.

$\mathrm{Na}$ análise dos dados foram utilizados 1000 intervalos RR consecutivos e foi feita uma filtragem digital complementada por manual, para eliminação de batimentos ectópicos prematuros e artefatos, e somente séries com mais de $95 \%$ de batimentos sinusais foram incluídas no estudo [5]. Estatística descritiva foi utilizada para análise e apresentação dos dados.

\section{Resultados}

Na Tabela 2 apresentamos os resultados estatísticos (média \pm desvio padrão) dos índices não-lineares descritos na Seção 2.

Tabela 2: Valores médios, seguidos dos respectivos desvios padrões, obtidos para os índices não-lineares (SD1, SD2, Entropia Aproximada, Entropia da Amostra, DFA e seus índices $\alpha_{1}$ e $\alpha_{2}$, Dimensão de Correlação, Expoente de Lyapunov e Conjectura de Kaplan-York) das séries RR experimentais dos voluntários estudados.

\begin{tabular}{|c|c|}
\hline Índices & Valores obtidos \\
\hline \hline Entropia Aproximada & $1,615 \pm 0,1367$ \\
\hline Entropia da Amostra & $1,617 \pm 0,3675$ \\
\hline DFA - $\alpha_{1}$ & $0,815 \pm 0,2310$ \\
\hline DFA - $\alpha_{2}$ & $0,7912 \pm 0,1279$ \\
\hline Dimensão de Correlação & $0,6835 \pm 1,3869$ \\
\hline Maior Expoente de Lyapunov & $0,12675 \pm 0,0658$ \\
\hline Conjectura de Kaplan-York & $1,17628 \pm 0,0869$ \\
\hline SD1 & $16,36 \pm 8,62$ \\
\hline SD2 & $26,64 \pm 11,93$ \\
\hline
\end{tabular}

Na Figura 2 apresentamos o Gráfico da Função de Autocorrelação, o Gráfico de Poincaré, o Plot de Recorrência e o gráfico obtido da Reconstrução do Espaço de 
Fase, de um dos voluntários, tomado como representante dentre os dez voluntários do grupo estudado. Os demais voluntários apresentaram o mesmo padrão gráfico.

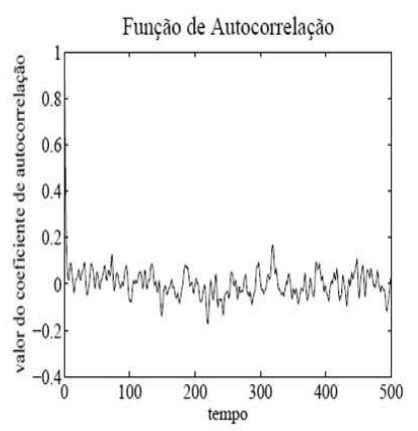

(a)

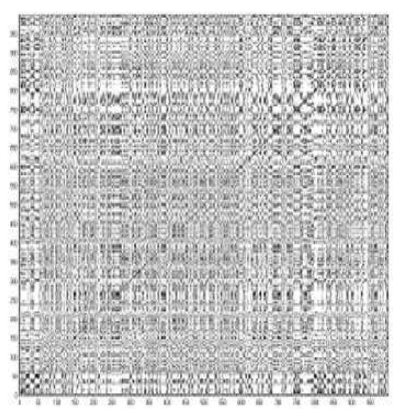

(c)

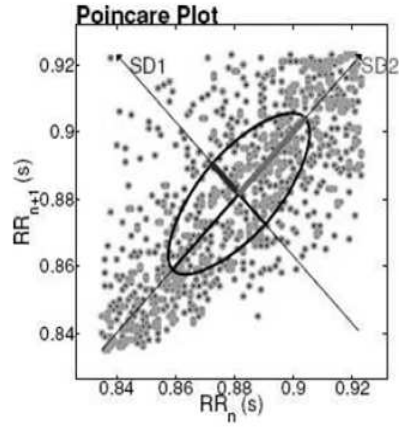

(b)

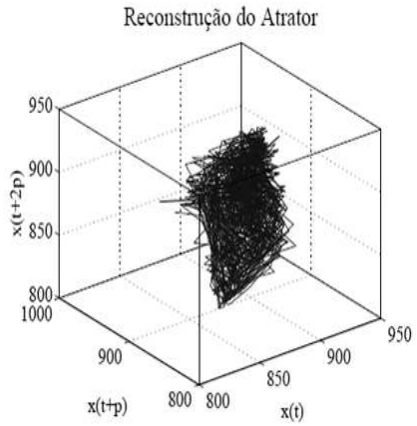

(d)

Figura 2: Gráficos relativos à VFC de um voluntário tomado como representante dentre os dez indivíduos da amostra estudada: (a) Função de Autocorrelação; (b) Gráfico de Poincaré; (c) Plot de Recorrência; (d) Reconstrução do Espaço de Fase.

\section{Discussão}

A entropia aproximada e da amostra apresentam-se moderadamente altos e condizem com o que é descrito na literatura, confirmando a característica de um grupo de indivíduos que apresentam séries temporais complexas e com alta variabilidade $[1,7,14]$. Além disso, os valores obtidos para os índices $\alpha_{1}$ e $\alpha_{2}$ estão próximos da unidade e temos $\alpha_{1}>\alpha_{2}$, o que também é uma característica própria de indivíduos 
saudáveis, de acordo com $[1,6]$.

A dimensão de correlação apresentou-se muito baixa, não estando de acordo com a literatura estudada $[1,6,7,14]$, enquanto que o expoente de Lyapunov, forneceu pelo menos um valor com sinal positivo, indicando o possível comportamento caótico da série temporal.

Quanto à conjectura de Kaplan-York, o fato de seu valor ser diferente de zero, por si só, já sugere a presença de um atrator associado a série temporal.

O índice SD1, o qual indica a influência do ramo parassimpático [14], é menor do que o valor do índice SD2, o qual indica a variabilidade global.

Com relação aos gráficos obtidos, todos os voluntários analisados apresentaram o mesmo padrão, mostrado na Figura 2. A função de autocorrelação (Figura 2(a)), apresenta oscilações tendendo à zero, o que fornece indícios de uma série temporal com possível comportamento caótico. O gráfico de Poincaré (Figura 2(b)), apresenta seus pontos dispersos, o que indica a complexidade do sistema cardíaco e sua facilidade a adaptar-se a mudanças abruptas ocorridas no meio ambiente. Quanto ao Plot de Recorrência (Figura 2(c)), podemos notar a inexistência de regiões em branco e a ausência de quadrados bem definidos, indicando uma alta VFC para este grupo de indivíduos. Na Figura 2(d) apresentamos o padrão do atrator reconstruído para a série RR, utilizando-se o método de Takens com dimensão de imersão igual a 3 e atraso 1, o qual ilustra o padrão do atrator associado a séries temporais da FC de indivíduos saudáveis.

\section{Conclusão}

Os resultados obtidos com o estudo de sinais unidimensionais emitidos pelo coração de indivíduos jovens saudáveis e sua análise por métodos não-lineares, permitiram caracterizar um possível comportamento caótico presente nas séries RR destes indivíduos.

Abstract. The heart rate variability (HRV) analysis is a technique used in the evaluation of the autonomous nervous system behavior that has been emerging as a simple noninvasive measure of the autonomous impulses. In this work we use techniques from the theory of nonlinear dynamical systems, including the chaos theory, in the study of the heart rate time series of healthy young people aiming to characterize the possible chaotic behavior of their HRV. The RR intervals time series of ten young healthy people, whose age range from 18 to 22 years, were analyzed. The calculated nonlinear indexes indicate the possible chaotic behavior of the analyzed series.

\section{Referências}

[1] U.R. Acharya, K.P. Joseph, N. Kannathal, C.M. Lim, J. S. Suri, Heart rate variability: a review, Medical and Biological Engineering, 44 (2006), 10311051. 
[2] G.P. Box, G.M. Jenkins, G.C. Reinsel, "Time series analysis. Forecasting and Control". Fourth Edition. John Wiley \& Sons, New Jersey, 2008.

[3] J.P. Eckmann, S. Oliffson Kamphorst, D. Ruelle, S. Ciliberto, Liapunov exponents from time series, Physical Review A, 34 (1986), 4971-4979.

[4] N. Fiedler-Ferrara, C.P.C. do Prado, "Caos: Uma Introdução", Editora Edgard Blücher Ltda., São Paulo, 1994.

[5] M.F. Godoy, I.T. Takakura, P. R. Correa, Relevância da análise do comportamento dinâmico não-linear (Teoria do Caos) como elemento prognóstico de morbidade e mortalidade em pacientes submetidos à cirurgia de revascularização miocárdica, Arq. Ciênc. Saúde, 12 No. 4 (2005), 167-71.

[6] F. Lombardi, Chaos theory, heart rate variability and arrhythmic mortality, Circulation, 101 (2000), 8-10.

[7] I. Macejark, The use of heart rate variability in cardiology, Bratisl Lek Listy, 103 (2002), 368-377.

[8] N. Marwan, N. Wessel, U. Meyerfeldt, A. Schirdewan, J. Kurths, Recurrenceplot-based measures of complexity and their application to heart-ratevariability data, Physical Review, 66 (2002), 1-8.

[9] L.G.G. Porto, L.F. Junqueira Júnior, Comparison of time-domain short-term heart interval variability analysis using a wrist-worn heart rate monitor and the conventional electrocardiogram, PACE, 32 (2009), 43-51.

[10] F. Takens, "Detecting strange attractors in turbulence", In: Dynamical systems and Turbulence, Lecture Notes in Mathematics, Vol. 898 pp. 366-381, SpringerVerlag, Berlin, 1981.

[11] Task Force of the European Society of Cardiology and the North American Society of Pacing and Electrophysiology, Heart rate variability: standards of measurement, physiological interpretation and clinical use, Circulation, 93, No. 5 (1996), 1043-1065.

[12] L.C.M. Vanderlei, C.M. Pastre, R.A. Hoshi, T.D. Carvalho, M.F. Godoy, Noções básicas de variabilidade da frequência cardíaca e sua aplicabilidade clínica, Rev Bras Cir Cardiovasc, 24, No. 2 (2009), 205-217.

[13] L.C.M. Vanderlei, R.A. Silva, C.M. Pastre, F.M. Azevedo, M.F. Godoy, Comparison of the Polar S810i monitor and the ECG for the analysis of heart rate variability in the time and frequency domains, Braz J Med Biol Res, 41 (2008), 854-859.

[14] C.D. Wagner, P.B. Persson, Chaos in the cardiovascular system: an update, Cardiovascular Research, 40 (1998), 257-264. 